\title{
A new class of rigid multi(azobenzene) switches featuring electronic decoupling: unravelling the isomerization in individual photochromes
}

\author{
Agostino Galanti, ${ }^{+a}$ Jasmin Santoro, ${ }^{t b}$ Rajesh Mannancherry, ${ }^{c}$ Quentin Duez, ${ }^{e}$ Valentin Diez-Cabanes, ${ }^{f}$ Michal \\ Valášek, ${ }^{b}$ Julien De Winter, ${ }^{e}$ Jérôme Cornil, ${ }^{f *}$ Pascal Gerbaux, ${ }^{e *}$ Marcel Mayor, ${ }^{b, c, d *}$ Paolo Samoria*
}

a Université de Strasbourg, CNRS, ISIS UMR 7006, 8 allée Gaspard Monge, 67000 Strasbourg, France. E-mail: samori@unistra.fr

${ }^{b}$ Karlsruhe Institute of Technology KIT, Institute of Nanotechnology, P.O. Box 3640, 76021 Karlsruhe, Germany.

${ }^{\mathrm{C}}$ Department of Chemistry, University of Basel, St. Johannsring 19, 4056 Basel, Switzerland. E-mail: marcel.mayor@unibas.ch

${ }^{d}$ Lehn Institute of Functional Materials (LFM), School of Chemistry, Sun Yat-Sen University (SYSU), Guangzhou 510275, China.

${ }^{\mathrm{e}}$ Organic Synthesis and Mass Spectrometry Laboratory, University of Mons, Place du Parc 20, 7000 Mons, Belgium. E-mail: pascal.gerbaux@umons.ac.be

${ }^{f}$ Laboratory for Chemistry of Novel Materials, University of Mons, Place du Parc 20, 7000 Mons, Belgium. E mail: jerome.cornil@umons.ac.be

\begin{abstract}
We report a novel class of star-shaped multi-azobenzene photoswitches comprising individual photochromes connected to a central trisubstituted 1,3,5-benzene core. The unique design of such $C_{3}$-symmetric molecules, consisting of conformationally rigid and pseudo-planar scaffolds, made it possible to explore the role of electronic decoupling in the isomerization of the individual azobenzene units. The design of our tris-, bis- and mono(azobenzene) compounds limits the $\pi$-conjugation between the switches belonging to the same molecule, thus enabling their efficient and independent isomerization of each photochrome. An in-depth experimental insight by making use of different complementary techniques such as UV-Vis absorption spectroscopy, high performance liquid chromatography and advanced mass spectrometry methods as ion mobility revealed an almost complete absence of electronic delocalization. Such evidence was further supported by both experimental (electrochemistry, kinetical analysis) and theoretical (DFT calculations) analyses. The electronic decoupling provided by this molecular design guarantees a remarkably efficient photoswitching of all azobenzenes, as evidenced by their photoisomerization quantum yields, as well as by the Z-rich UV photostationary states. Ion mobility mass spectrometry was exploited for the first time to study multi-photochromic compounds revealing the occurrence of a large molecular shape change in such rigid star-shaped azobenzene derivatives. In view of their high structural rigidity and efficient isomerization, our multi-azobenzene photoswitches can be used as key components for the fabrication of complex stimuli-responsive porous materials.
\end{abstract}

\section{INTRODUCTION}

Molecules and supramolecules that are capable to undergo structural rearrangements when subjected to an external stimulus have attracted a great deal of attention as they can represent the key building blocks for the fabrication of responsive molecular devices. ${ }^{1}$ Sophisticated dynamic functions are at the basis of many processes in Nature, ultimately ruling the most complex phenomena of life. Such a plethora of complex functions takes place in chemical systems which are able to respond to a variety of independent inputs including chemical, electrochemical and 
photochemical stimuli. ${ }^{2}$ By mimicking Nature, one among the greatest challenges in the realization of artificial switches and motors consists in the integration of multiple and independent responsive units in a single molecular system. ${ }^{3}$ Among various inputs, the use of photons has been regarded as one of the most promising for technological application thanks to the possibility of remote application with high spatiotemporal resolution, without generating waste products. ${ }^{4}$ Recently, increasing interest has been devoted to the design and synthesis of multi-photochromic architectures. ${ }^{5}$ This is particularly appealing since it allows to realize systems capable to toggle between multiple states (in principle $2^{\mathrm{n}}$ if all $n$ photochromic units are distinguishable) in contrast to conventional photochromes, which are typically bistable systems. ${ }^{6}$ The integration of more than one photochromic unit within the same backbone should allow to individually address each state by irradiation with light at different wavelengths, provided the isomers of each photochrome have distinct spectral features. Nevertheless, the development of orthogonally-responsive systems is still in an embryonal phase. ${ }^{3}$ Conversely, if all photochromes in the molecular backbone are equivalent, one could obtain a highly contrasted photoresponse. ${ }^{7}$ The implementation of multiple switches in the same covalent scaffold showed to be not a trivial task, since in order to guarantee the photoactivity of all units, interchromophore interactions such as conjugation or energy transfer must be avoided. ${ }^{6 a} 8$ Azobenzene is the most popular photochromic compound, and its celebrity is due to the large structure rearrangement occurring upon conversion from the $E$ to the $Z$ isomer, alongside with its photochemical robustness. ${ }^{9}$ Such characteristics make azobenzene particularly suitable as molecular-scale actuators. In order to exploit and/or amplify the shape variation of the switch to a greater length scale, it is vital to embed such a unit in a conformationally rigid molecular scaffold. ${ }^{10}$ Towards this end, a viable strategy consists of including them into rigid aromatic structures. However, such a solution typically prevents full or even partial photochromism of the system, since electron delocalization precludes the access to independent photoreaction pathways. ${ }^{11}$ A systematic research work was executed in order to find the ideal substitution pattern ensuring the coexistence of structural rigidity and satisfying photoswitching capacity; in this framework, little was done on systems containing more than one photochromic unit. ${ }^{12}$ In particular, star-shaped multi-azobenzene systems were realized by mutual chromophore connection to non-planar, ${ }^{12 a, 13}$ or to quasi-planar ${ }^{12 \mathrm{~b}-}$ ${ }^{d}$ cores. However, the isomerization behavior of the individual switches has been characterized only to a partial extent by means of ${ }^{1} \mathrm{H}-\mathrm{NMR}$ spectroscopy; in the case of mutual connection via a central amine linker, the undesirable presence of electronic delocalization among the chromophores was observed. ${ }^{12 a}$

Nonetheless, it is worth pointing out that the electronic decoupling between the photochromes is indeed a necessary target in order to preserve the photoactivity of such a multichromophoric system. ${ }^{10-11,14}$ Inspired by the shape of rigid and highly planar $C_{3}$-symmetrical aromatic systems, which have been successfully exploited as organic ligands for supramolecular coordination complexes and metal-organic frameworks, ${ }^{15}$ we designed and synthesized a simple symmetrical aromatic system containing up to three azobenzene units. Recently, we reported the tris(azobenzene) system 1 and explored its 2D self-assembly at the solid-liquid interface by in-situ scanning tunneling microscopy (STM) analysis. ${ }^{16}$ Such a system is composed by three azobenzene arms connected through a central benzene ring substituted in 1,3,5-positions. This substitution guarantees the absence of photochromism quenching, while the high rigidity imposed by the aromatic core offers large conformational changes upon isomerization. Here we chose to use carboxylic functions as they are the principal end group employed in organic building blocks for metal-organic frameworks; moreover such functional groups, thanks to their strong and highly directional H-bonding, are known to give rise to $2 \mathrm{D}$ and $3 \mathrm{D}$ self-assembled structures with peculiar topologies. ${ }^{17}$ Although this substitution generally is not found to be detrimental for the photochromic performance of azobenzene switches, we have decided to undertake a detailed study to demonstrate the effectiveness of our design. The demonstration of its photochromism requires an in-depth analysis on photoisomerization efficiency of each azobenzene unit, thereby unravelling the subtle details of inter-chromophoric interactions. This issue has never been addressed so far by combining different experimental methods to explore simultaneously the variation in the photophysical properties and volumetric/conformational changes upon isomerization.

In order to fully unveil the photochromism of each individual azobenzene embedded in a rigid multichromophoric scaffold, we have extended our study to a series of planar multi-azobenzene compounds having either one, two or three switches branching out in the 1,3,5-positions on a central benzene unit (Figure 1). To gain a comprehensive insight, detailed investigations on the photophysical properties and photoisomerization quantum yields performed by UV-Vis absorption spectroscopy have been combined to high performance liquid chromatography (HPLC) and ionmobility mass spectrometry (IMMS) to bestow information onto the photogenerated isomer mixtures. The electronic properties of such multiphotochromic switches were also investigated by means of cyclic voltammetry (CV) and 
differential pulse voltammetry (DPV). Both HPLC and IMMS were used to separate the four possible $(E, E, E),(E, E, Z)$, $(E, Z, Z)$ and $(Z, Z, Z)$ isomers of 1 upon UV light irradiation. On the one hand, HPLC was employed to determine the composition of the photostationary states (PSS) and to follow the $Z \rightarrow E$ thermal isomerization kinetics; by studying the temporal evolution of the isomer mixture, we could directly prove the independent kinetic behavior of each azobenzene unit embedded within the same molecular scaffold. On the other hand, IMMS provided unambiguous evidence for the large difference in shape of the aforementioned isomers, ${ }^{18}$ as a result of their structural rigidity. To shed further light onto the multi-photochromism and the shape variation of the various isomers, we have designed three novel star-shaped model compounds in which the diazene-1,2-diyl groups have been progressively substituted with similar (in contour length and rigidity) yet non-photoresponsive ethyn-1,2-diyl units, i.e. the bis(azobenzene) $\mathbf{2}$, mono(azobenzene) $\mathbf{3}$ as well as the derivative $\mathbf{5}$ exhibiting three 1,2-diphenylethyne (tolane) arms. As reference compounds, we have also investigated two linear azobenzene derivatives, namely extended 4-[(biphenyl-4yl)diazenyl]benzoic acid 4 and the commercially available 4-(phenyldiazenyl)benzoic acid 6 (Figure 1). Our investigation suggests that it is possible to design relatively small rigid molecules having efficient, fully independent (i.e. electronically decoupled) photochromic units by simply connecting them in the meta- position on a central benzene ring. Upon $E \rightarrow Z$ isomerization, the various isomers of compounds 1-3 show different shapes, as evidenced by the change in the collisional cross section (CCS) of their ions, a quantity that is directly correlated to the corresponding ion geometries in the gas phase. These evidences given by ion mobility mass spectrometry represent also the first investigation conducted on a multi-photochromic compound with this powerful technique. We could also show that the separated $Z$ isomer(s) of $\mathbf{1}$ could be converted to the thermodynamically stable all-(E) structure within the mass spectrometer by collisional heating in the gas phase.
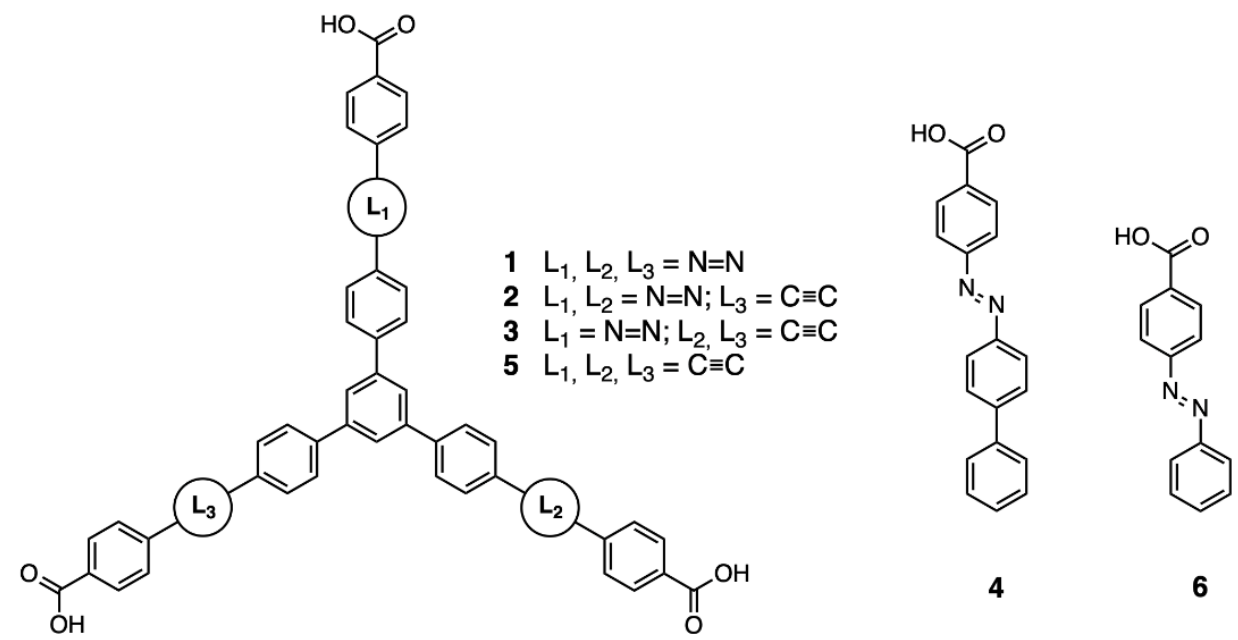

Figure 1. Structure formulae of the compounds employed in the present study.

\section{RESULTS AND DISCUSSION}

\section{Chemical synthesis}

Our synthetic strategy towards the four members of the star-shaped azobenzene family $\mathbf{1 - 3}$, and $\mathbf{5}$ is displayed in the Supporting Information (Schemes SI 1-4). While the synthesis of $C_{3}$-symmetric photoresponsive derivative 1 , as well as of its non-photoactive analogue 5 was already reported, ${ }^{16}$ both unsymmetrical derivatives 2-3 were stepwise assembled using Pd catalyzed Suzuki cross-coupling protocol between a three-directional core (e.g. 1,3,5tribromobenzene) and the corresponding arm moieties. Non-photoactive tolane arm was obtained by a sequence of Sonogashira coupling and Miyaura borylation, whereas azobenzene arms were assembled via the Mills reaction of aromatic amine with nitroso compound. A similar sequence of reactions was used for the preparation of the linear azobenzene derivative 4. Detailed synthetic protocols of all new compounds are provided in the Supporting Information and the identity of all compounds was corroborated by their full characterization based on ${ }^{1} \mathrm{H}$ - and ${ }^{13} \mathrm{C}$ NMR spectroscopy, mass spectrometry data and elemental analyses. 


\section{Photochemical characterization}

In a first instance, the photophysical properties of compounds 1-6 and the light-induced isomerization of azobenzenecontaining compounds have been investigated in DMSO with the addition of a base excess to ensure their complete solubilization. Particular attention was paid to such measurements, as they could provide the first evidence on the extent of electronic coupling between the photoswitchable moieties of our interest. Such properties are summarized in Table 1. The UV-Vis absorption spectra (Figure S 31) of all-(E) tris(azobenzene) compound 1 and its star-shaped bis(azobenzene) $\mathbf{2}$ and mono(azobenzene) $\mathbf{3}$ derivatives exhibit two characteristic transitions related to the azobenzene chromophore (Azo): a higher energy band due to the $\pi-\pi^{*}$ transition and a lower energy $n-\pi^{*}$ transition. For 1-3, the absorption maxima are located at $c a .370 \mathrm{~nm}$ for the $\pi-\pi^{*}$ and $c a .455 \mathrm{~nm}$ for the lower energy transition. When compared to the linear azobenzene chromophore 4, a slight bathochromic shift is observed (about $10 \mathrm{~nm}$ ), which together with an intensity increase (if compared to the absorption spectrum of 4 reported in $\varepsilon$ multiplied by a factor of three, Figure S 31) and a partial broadening, evidences that weak conjugation occurs between the arms of 1$\mathbf{3}$ despite the meta substitution, as previously reported on similar star-shaped systems. ${ }^{19}$ Compounds 2-3 display one additional transition located in the UV region $(c a .320 \mathrm{~nm}$ ) which can be ascribed to the 1,2-diphenylethyne chromophore unit (PE), as shown by comparison with the $C_{3}$-symmetrical non-photochromic derivative $\mathbf{5}$. Despite the existence of partial conjugation between the chromophores in our star-shaped systems, an additive behavior of the absorption spectra of the single chromophores is evident, by observing the $\varepsilon$ values of the most intense transitions of Azo and PE units (Table 1), which enlightens that the transitions are substantially localized on each chromophore unit. This is clearly revealed by observing that the maxima of Azo-centered transitions are constant between 1, 2 and $\mathbf{3}$. Moreover, the model linear Azo chromophore 4 indicates that the $\lambda_{\max }$ related to the $\pi-\pi^{*}$ transition is located at $c a$. $360 \mathrm{~nm}$, a wavelength which should be compared to the known position of the same band for azobenzene (ca. 315 $\mathrm{nm}^{9 \mathrm{a}}$ ) and 4-(phenyldiazenyl)benzoic acid 6 (ca. $335 \mathrm{~nm}$ ); such a red shift can therefore be attributed to the extension of the conjugated system by including one additional phenylene unit in the para-position with respect to the azobenzene moiety.

Table 1. Photochemical characterization of compounds 1-6 in air-equilibrated DMSO (+ exc. NaOH) at $298 \mathrm{~K}{ }^{\text {a }}$

\begin{tabular}{|c|c|c|c|c|c|c|c|c|c|}
\hline & $\lambda_{\max }$ all-(E & omer $[\mathrm{nm}$ & ${ }^{3}\left[\mathrm{~L} \mathrm{~mol}^{-1} \mathrm{cr}\right.$ & $\Phi_{E}$ & & $\Phi_{z}$ & & UV-PSS & Vis - PSS \\
\hline & $\pi-\pi^{*} \mathrm{PE}$ & $\pi-\pi^{*}$ Azo & $\mathrm{n}-\pi^{*}$ Azo & Overall & $\begin{array}{c}\text { Single } \\
\text { Azo }\end{array}$ & Overall $^{e}$ & $\begin{array}{c}\text { Single } \\
\text { Azo }\end{array}$ & composition [\%] ${ }^{d}$ & composition [\%] \\
\hline & & & & & & & & $55(Z, Z, Z)$ & $9(Z, Z, Z)$ \\
\hline & & & & & & & & $27(E, Z, Z)$ & $17(E, Z, Z)$ \\
\hline 1 & - & $370(110)$ & 455 (5.7) & 3.3 & 8.8 & 28 & 77 & $13(E, E, Z)$ & $30(E, E, Z)$ \\
\hline & & & & & & & & $5(E, E, E)$ & $44(E, E, E)$ \\
\hline & & & & & & & & $61(z, Z)$ & $13(Z, Z)$ \\
\hline 2 & $320(65)$ & $370(77)$ & 455 (3.7) & 4.5 & 9.5 & 47 & 98 & $29(E, Z)$ & $32(E, Z)$ \\
\hline & & & & & & & & $10(E, E)$ & $55(E, E)$ \\
\hline 3 & $320(100)$ & $370(42)$ & $455(1.8)$ & 91 & & 100 & & $73(Z)$ & $32(Z)$ \\
\hline & $320(100)$ & $3 / 0(42)$ & 455 (1.8) & 9.1 & & 100 & & $27(E)$ & $68(E)$ \\
\hline 5 & $320(130)$ & - & - & - & & - & & & \\
\hline 4 & - & $360(30)$ & 455 (1.4) & 9.6 & & 88 & & & \\
\hline 6 & - & $335(21)$ & $450(0.8)$ & 11 & & 70 & & & \\
\hline
\end{tabular}

${ }^{a}$ Excess $\mathrm{NaOH}$ (6.0 eq. for 1-3 and 5, 2.0 eq. for 4,6). ${ }^{\mathrm{b}} \mathrm{UV} \pi-\pi^{*}$ irradiation, $\lambda_{\text {irr }}=367 \mathrm{~nm}$ (FWHM $\left.=9 \mathrm{~nm}\right) .{ }^{\mathrm{c}}$ Vis $\mathrm{n}-\pi^{*}$ irradiation, $\lambda_{\text {irr }}=451 \mathrm{~nm}(\mathrm{FWHM}=$ $20 \mathrm{~nm}$ ). d Determined by HPLC-MS. e Overall isomerization quantum yield, calculated using the $\varepsilon$ of the compounds (see text). ${ }^{\mathrm{f}}$ Isomerization quantum yield of the single chromophore unit, calculated using the $\varepsilon$ of the linear azobenzene compound $\mathbf{4}$ (see text).

The photoisomerization of compounds 1-4 and 6 was studied upon irradiation with UV $\left(\lambda_{\max }=367 \mathrm{~nm}\right)$ and Vis $\left(\lambda_{\max }=\right.$ $451 \mathrm{~nm}$ ), while proof of the non-photoreactivity of $\mathbf{5}$ was already given in previous studies. ${ }^{16} \mathrm{UV}$ irradiation of the aforementioned compounds in DMSO solution showed spectral variations typical for $E \rightarrow Z$ isomerization of azobenzene derivatives (Figure 2), with a progressive decrease in the absorbance in the $(E)$-Azo $\pi-\pi^{*}$ region, accompanied by an increase in the absorbance in the higher energy UV region, together with the same variation in the visible range. Both evidences are related to a decrease in concentration of the (E)-isomer(s) accompanied by an obvious increase in the $(Z)$ ones upon reaching the photostationary state (UV - PSS). The initial spectral features were seen to recover partially upon subsequent Vis irradiation reaching another PSS (Vis - PSS), while complete recovery was encountered only by storing the solution in the dark over several days. It is crucial to note for all compounds, 
especially for tris(azobenzene) $\mathbf{1}$ and bis(azobenzene) 2, the presence of clear isosbestic points throughout both $E \rightarrow Z$ and $Z \rightarrow E$ photoconversions, which provide unambiguous evidence for the absence of inter-chromophore interactions. ${ }^{11}$ The composition of the UV - and Vis - PSS was characterized by HPLC (Table 1): thanks to the known difference in molecular dipole moment of the $E$ and $Z$ isomers of azobenzenes, all possible isomeric forms of 1-3 could be separated by employing this technique, thus proving the formation of all the expected isomers for the multiazobenzene compounds (qualitative absorption spectra in Figure 2, a, b insets, Table 1). The absorption spectra of single isomers obtained by HPLC are also nicely comparable with the spectra simulated at a TD-DFT level (see details on methods and Figure S 37 in the Supporting Information). It is worth noting that for both the tris(azobenzene) 1 and bis(azobenzene) $\mathbf{2}$ derivatives, it is possible to reach a (Z)-rich UV - PSS (Table 1, quantified by HPLC-MS, see the Supporting Information for experimental details). The efficiency of $E \rightarrow Z$ and $Z \rightarrow E$ photoreactions was estimated by evaluating their quantum yields $(\Phi)$. The values given in Table 1 and indicated as "overall" $\Phi$ were calculated by using the $\varepsilon$ of each compound; therefore, they refer to the quantum yield of isomerization of all azobenzene units within each molecular scaffold, while the "single Azo" values were calculated using the $\varepsilon$ of the linear azobenzene 4, and provide a comparative estimation of the $\Phi$ of the single photochrome. ${ }^{20}$ It is particularly interesting to benchmark the "single azo" $\Phi$ of star-shaped compounds 1-3 with standard azobenzene derivatives, since it is generally known that conjugation between the chromophores drastically lowers the photoreaction efficiency. ${ }^{11 \mathrm{~b}}$ We will take azobenzene as benchmark, showing for $E \rightarrow Z$ isomerization $\Phi \approx 0.14-0.15$ in polar solvents $\left(\lambda_{\text {irr }} \approx 345 \mathrm{~nm}\right) ;{ }^{11 \mathrm{~b}, 21}$ in comparison, 4(phenyldiazenyl)benzoic acid 6 shows a slightly lower value $(\Phi \approx 0.11$ ) due to the presence of the electronwithdrawing carboxyl moiety in the para-position with respect to the chromophore. ${ }^{20}$ Extension of the $\pi$-conjugated backbone in $\mathbf{4}$ is accompanied by an almost negligible reduction $(\Phi \approx 0.10)$ of efficiency for the process; interestingly, for the star-shaped mono(azobenzene) derivative 3, $\Phi \approx 0.09$ and for both multi-azobenzene scaffolds 2 and 1 , the "single Azo" $\Phi$ exhibits the same value (Table 1). Conversely, for $Z \rightarrow E$ photoreaction induced by Vis irradiation, the quantum yields of $\mathbf{2}$ and $\mathbf{1}$ (Table 1 ) are even higher than azobenzene (for the latter, $\Phi \approx 0.63, \lambda_{\text {irr }} \approx 436 \mathrm{~nm}$ ) itself. ${ }^{22}$ However, the values obtained from these experiments are expected to display a higher uncertainty, since the concentration of the (E)-isomer(s) in the initial conditions (UV - PSS) was not zero. ${ }^{23}$
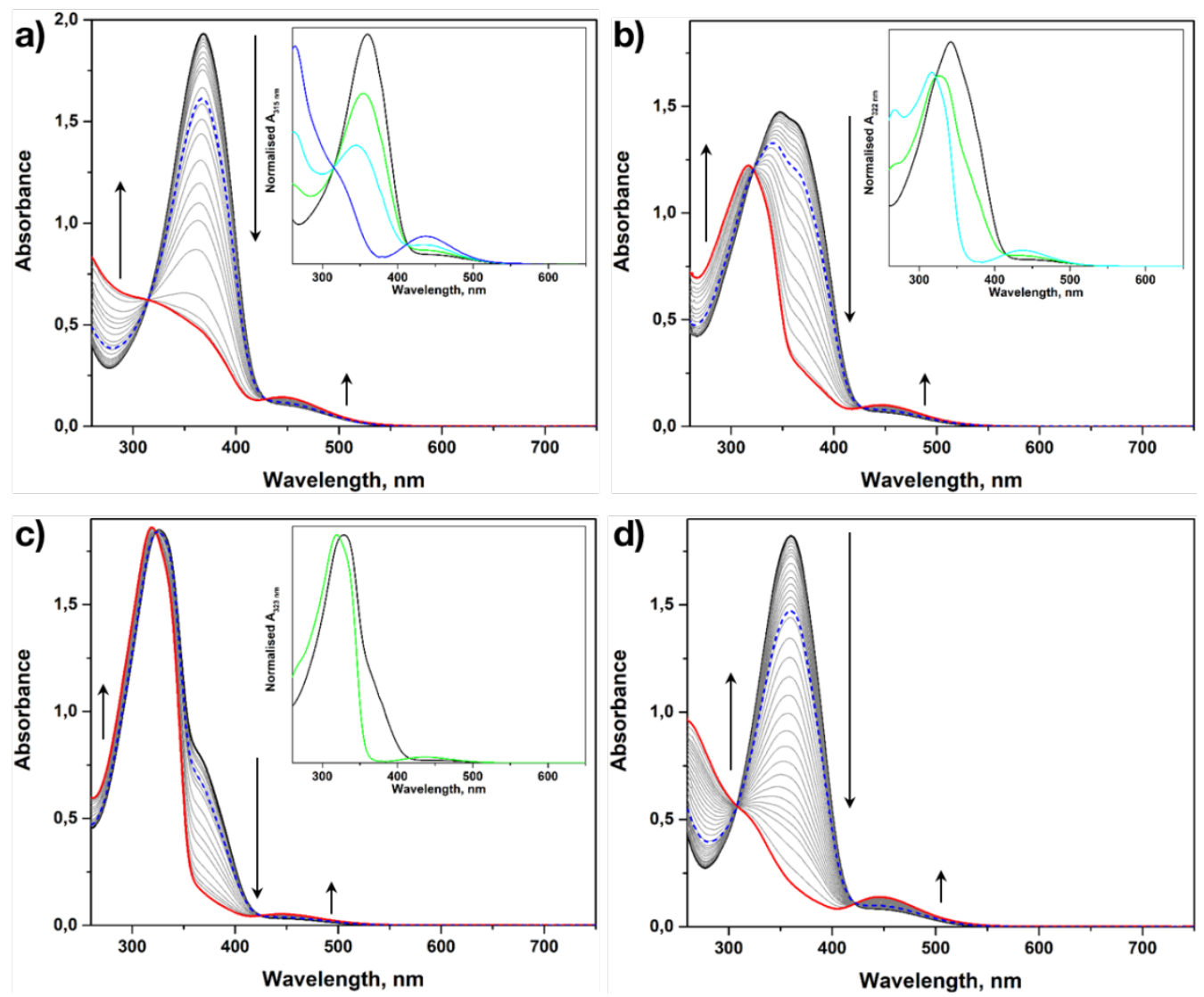

Figure 2. UV-Vis spectral variation upon UV irradiation in DMSO with an excess of base: black line, no irradiation, red line UV PSS, blue dotted line Vis PSS. a) Compound 1, $\mathrm{c}=2.0 \times 10^{-5} \mathrm{M}$ inset, absorption spectra of the single isomers recorded upon HPLC 
separation black line $(E, E, E)-\mathbf{1}$, green line $(E, E, Z)-\mathbf{1}$, light blue line $(E, Z, Z)-\mathbf{1}$, dark blue line $(Z, Z, Z)-\mathbf{1}, \mathrm{b}) \mathbf{2}, \mathrm{c}=2.0 \times 10^{-5} \mathrm{M}$ inset, black line $(E, E)-2$, green line $(E, Z)-\mathbf{2}$, light blue line $(Z, Z)-2, c) 3, c=2.0 \times 10^{-5} \mathrm{M}$ inset, black line $(E)-3$, green line $\left.(Z)-3, d\right) 4, c=6.0 \times 10^{-5} \mathrm{M}$.

\section{Electrochemistry}

The redox properties of azobenzene have been widely studied, and there is general agreement on the mechanism of its electrochemical reduction in aprotic media. ${ }^{24}$ Cyclic voltammetry (CV) has been successfully employed to obtain a qualitative insight into the extent of the $\pi$-conjugation of multi-azobenzene systems by focusing on their first reduction reaction. ${ }^{11}$ Here, we evaluated the electrochemical properties of compounds 1-6 together with azobenzene as reference in dry DMF, using tetra- $n$-butylammonium hexafluorophosphate $(c=0.1 \mathrm{M})$ as supporting electrolyte in a comparative fashion by means of cyclic voltammetry and differential pulse voltammetry (DPV, Figure S 34-36, potentials reported vs. ferrocene redox couple, see Supporting Information for detailed description, procedure and experimental data). In order to obtain a correct assignment of the redox processes visualized in the experiments, we compared the voltammograms of 1-6 with azobenzene in our experimental conditions. All potentials expressed here have been determined at the peaks of the DPV experiments. Azobenzene shows a quasi-reversible reduction peak at $1.77 \mathrm{~V}$, with a spacing between the cathodic and anodic peaks $\left(\Delta E_{p}\right)$ of $70 \mathrm{mV}$, coherently with what is reported in the literature (Figure S 36). In all cases, the voltammograms of compounds 1-6 owing carboxylic acid functionalities showed a complex redox behavior, which we assigned to the subsequent reduction of the carboxylic fragment and diazene moiety (when present, Figure S 34-36). Compound 6 presents a quasi-reversible redox reaction at $-1.81 \mathrm{~V}\left(\Delta E_{p}\right.$ $=70 \mathrm{mV}$ from CV) associated to the reduction of the azobenzene unit (Figure S 36). Analogously, compound 4 shows the redox reaction on the diazo moiety occurring at $-1.76 \mathrm{~V}\left(\Delta E_{p}=80 \mathrm{mV}\right.$ from CV, Figure S 34); the decrease in energy for its reduction is coherent with the extension of its $\pi$-conjugated core, while the slight broadening indicates a slightly lower reversibility. Interestingly, the electrochemical behavior of $\mathbf{1}$ demonstrated to be analogous to $\mathbf{4}$ and $\mathbf{6}$, except for its broader character (Figure S 34, $\Delta E_{p}>150 \mathrm{mV}$ for Azo reduction, from CV). Importantly, DPV clearly shows only one peak for the reduction of the azobenzene units, centered at $-1.75 \mathrm{~V}$, i.e., at a potential comparable to the value obtained from 4. The latter, together with the absence of any splitting for this electrochemical process, gives further evidence for the efficient electronic decoupling between the photochromic units obtained with our molecular design. ${ }^{11 a}$ The bis(azobenzene) $\mathbf{2}$ and mono(azobenzene) $\mathbf{3}$ star-shaped derivatives display broad CV curves (Figure $\mathrm{S}$ $35)$, in which the redox behavior of the azobenzene unit(s) is overshadowed by the irreversible reaction occurring on the carboxylic moieties (Figure S 34). Nevertheless, using DPV, it is possible to identify the reduction peak of the Azo units on 2 at $-1.75 \mathrm{~V}$, hence occurring at potential analogous as for $\mathbf{1}$ and $\mathbf{4}$. By and large, the above-mentioned experiments, highlighting that the electrochemical reduction of azobenzene units in multiphotochromic derivatives 1 and $\mathbf{2}$ occur at the same redox potential, give further evidence for their electronic decoupling.

\section{Kinetics of $Z \rightarrow E$ thermal isomerization}

The $Z \rightarrow E$ thermal reactions for 1-3 were followed by HPLC in order to monitor the population of each isomer over time starting from the UV - PSS. For the sake of comparison, the thermal back-isomerization for $\mathbf{4}$ was studied by UVVis absorption spectroscopy. Multi-azobenzene compounds are known to undergo isomerization from the all-(Z) to the all- $(E)$ forms via the mixed $(Z, E)$ isomers, and the reaction mechanism can be treated as a consecutive and irreversible cascade reaction involving first-order reactions. ${ }^{11 a}, 25$ Upon plotting the isomer fraction over time (Figure $S$ 42-56), we could fit the evolution curves with the suitable kinetic equations (detailed procedure, equations and experimental data in the Supporting Information), thus obtaining the reaction rates for each azobenzene unit (Table $S$ 2). The activation energy parameters for the thermal isomerization were determined by Eyring analysis (Error! Reference source not found.). Such evidences made it possible to prove that for our tris(azobenzene) system 1, the mechanism of the thermal reaction follows the expected pseudo-first order cascade reaction: $(Z, Z, Z)-\mathbf{1} \rightarrow(E, Z, Z)-\mathbf{1} \rightarrow$ $(E, E, Z)-\mathbf{1} \rightarrow(E, E, E)-\mathbf{1}$. The bis(azobenzene) 2: $(Z, Z)-\mathbf{2} \rightarrow(E, Z)-\mathbf{2} \rightarrow(E, E)-\mathbf{2}$ exhibits equivalent behavior. For such cascade reactions, it is necessary to account for the statistical character of the first reaction(s), since the first isomerization could occur on three equivalent azobenzene units for $\mathbf{1}$, and on two for molecule $\mathbf{2}$. In our case, the normalized rate constant $\left|k_{1}\right|$ for the $(Z, Z, Z)-1 \rightarrow(E, Z, Z)-1$ reaction was divided by three. Similarly, the normalized rate constant $\left|k_{2}\right|$ accounting for $(E, Z, Z)-\mathbf{1} \rightarrow(E, E, Z)-\mathbf{1}$ and $(Z, Z)-\mathbf{2} \rightarrow(E, Z)-\mathbf{2}$ reactions was divided by two. It is worth mentioning that, for 
both the tris(azobenzene) $\mathbf{1}$ and bis(azobenzene) $\mathbf{2}$, the thermal isomerization rate constants are substantially analogous, thus indicating that the azobenzene units do not influence one another; they are electronically and geometrically decoupled, thus explaining the absence of cooperative effects. Since the azobenzene units are reciprocally connected by a rigid phenylene spacer, complete electronic decoupling could not be postulated only based on the electronic spectra of compounds 1-3 (see above). Those in fact enlighten the occurrence of partial $\pi$ conjugation between the azobenzene chromophores when embedded in the $\pi$-extended star-shaped system, when compared to 4 . Indeed, the examination of the activation energy parameters for the thermal $Z \rightarrow E$ isomerization obtained under dark revealed that all azobenzene units in compounds $\mathbf{1}$ and $\mathbf{2}$ display the same kinetic behavior; interestingly, the reference star-shaped mono(azobenzene) $\mathbf{3}$ displays analogous rate constants and activation energy parameters (Table 2 and Table S 2). Reference compound $\mathbf{4}$ instead shows slightly faster thermal isomerization, if compared to the star-shaped compounds 1-3 (Error! Reference source not found. and Table S 2). The values are however within the same order of magnitude and are comparable with similar rigid azobenzene and bis(azobenzene) derivatives found in the literature. ${ }^{13 a}$ Here, it is visible how the mild electron-withdrawing character of the carboxylic group in the para-position with respect to the azobenzene moiety does not sensibly lower the activation energy for the thermal isomerization, in contrast to stronger electron acceptor groups involving mesomeric effects (e.g. nitro $\mathrm{NO}_{2}$ or cyano $\left.-\mathrm{CN}\right) .{ }^{26}$

Table 2. Kinetic data for the thermal $Z \rightarrow E$ isomerization of compounds 1-4.

$k\left[\mathrm{~s}^{-1}\right]^{\mathrm{b}} \quad \Delta G^{\neq \mathrm{b}}\left[\mathrm{kJ} \mathrm{mol}^{-1}\right] \quad \Delta H^{\neq}\left[\mathrm{kJ} \mathrm{mol}^{-1}\right] \quad \Delta S^{\neq}\left[\mathrm{J} \mathrm{mol}^{-1}\right] \quad \Delta E_{a}^{\neq}\left[\mathrm{kJ} \mathrm{mol}{ }^{-1}\right]$

\begin{tabular}{|c|c|c|c|c|c|c|c|c|c|c|c|c|c|c|c|}
\hline & $\left|k_{1}\right|$ & $\left|k_{2}\right|$ & $k_{3}$ & $\left|\Delta \boldsymbol{G}_{1}^{\neq}\right|$ & $\left|\Delta \boldsymbol{G}_{2}^{\neq}\right|$ & $\Delta \boldsymbol{G}_{3}^{\neq}$ & $\left|\Delta H_{1}^{\neq}\right|$ & $\left|\Delta \boldsymbol{H}_{2}^{\neq}\right|$ & $\Delta \boldsymbol{H}_{3}^{\neq}$ & $\left|\Delta S_{1}^{\neq}\right|$ & $\left|\Delta S_{2}^{ \pm}\right|$ & $\Delta \boldsymbol{S}_{3}^{\neq}$ & $\left|\Delta E_{a 1}^{ \pm}\right|$ & $\left|\Delta E_{a 2}^{ \pm}\right|$ & $\Delta E_{a 3}^{ \pm}$ \\
\hline 1 & $\begin{array}{l}3.8 x \\
10^{-6}\end{array}$ & $\begin{array}{l}3.7 \times \\
10^{-6}\end{array}$ & $\begin{array}{l}3.9 \times \\
10^{-6}\end{array}$ & 104 & 104 & 104 & 88 & 89 & 88 & -55 & -52 & -52 & 90 & 91 & 91 \\
\hline 2 & - & $\begin{array}{l}3.5 \times \\
10^{-6}\end{array}$ & $\begin{array}{l}4.2 \times \\
10^{-6}\end{array}$ & - & 104 & 104 & - & 90 & 88 & - & -48 & -54 & - & 92 & 90 \\
\hline 3 & - & - & $\begin{array}{l}3.8 \times \\
10^{-6}\end{array}$ & - & - & 104 & - & - & 90 & - & - & -47 & - & - & 92 \\
\hline $4^{c}$ & - & - & $\begin{array}{l}1.1 X \\
10^{-5}\end{array}$ & - & - & 102 & - & - & 86 & - & - & -52 & - & - & 88 \\
\hline
\end{tabular}

aParameters with appendix $\mathrm{x}_{1}$ refer to $(Z, Z, Z)-\mathbf{1} \rightarrow(E, Z, Z)-\mathbf{1}$ isomerization, and those with $\mathrm{x}_{2}$ to $(E, Z, Z)-\mathbf{1} \rightarrow(E, E, Z)-\mathbf{1}$ and $(Z, Z)-\mathbf{2} \rightarrow(E, Z)-\mathbf{2}$ and $x_{3}$ to $(E, E, Z)-\mathbf{1} \rightarrow(E, E, E)-\mathbf{1},(E, Z)-\mathbf{2} \rightarrow(E, E)-\mathbf{2}$ and $(Z)-\mathbf{3}-\mathbf{4} \rightarrow(E)-\mathbf{3}-\mathbf{4}$. The normalized rate constants $\left|k_{1}\right|$ and $\left|k_{2}\right|$ were divided by three or two in order to account for the statistical character of the $(Z, Z, Z)-\mathbf{1} \rightarrow(E, Z, Z)-\mathbf{1},(E, Z, Z)-\mathbf{1} \rightarrow(E, E, Z)-\mathbf{1}$ and $(Z, Z)-\mathbf{2} \rightarrow(E, Z)-\mathbf{2}$ isomerizations, respectively. ${ }^{\mathrm{b}}$ At $298 \mathrm{~K}$. 'Determined by UV-Vis absorption spectroscopy.

\section{Quantum-chemical modeling}

DFT calculations give us access to the electronic and optical properties of the single molecules in their different isomers (see Table 3, and further details in Supporting Information). Therefore, we can analyze the degree of decoupling between the different chromophores by comparing the properties of the full $(E)$ and $(Z)$ isomers of compound $\mathbf{1}$ with the isomers of the reference compound 4, as already done at the experimental level in the first two sections. A first factor which reduces the coupling between the different units is the non-planarity of the $\pi$-backbone. The steric hindrance between the $\mathrm{H}$ atoms of the central phenyl ring and those of the adjacent rings results in a dihedral angle $\vartheta$ (see Figure S 38) between these rings of about $38^{\circ}$ in all studied compounds. Note that the excitonic coupling between the different photochromic branches (measured as the splitting of $\pi-\pi^{*} / n-\pi^{*}$ transitions in $(E, E, E)-1$ and $(Z, Z, Z)-1$ isomers, see Figure $S 40)$ is considerably reduced (31\%) if compared with the planar structures due to the steric hindrance (see Figure S41). Regarding the electronic properties, the similar energy gaps of the reference compound $\mathbf{4}$ with respect to compound $\mathbf{1}$ (3.47 eV for both $(E)-\mathbf{4}$ and $(E, E, E)-\mathbf{1} ; 3.51$ vs $3.48 \mathrm{eV}$ for $(Z)-\mathbf{4}$ and $(Z, Z, Z)-\mathbf{1}$, see Table 3) show that there is no major increase in the conjugation when connecting the different chromophores to the central ring. At the optical level, the $n-\pi^{*}$ transitions of $(Z)$ isomers present very similar $\lambda_{\max }$ values in all compounds. In contrast, for the $\pi-\pi^{*}$ transitions of the $(E)$ isomers, there is a small red shift (11 nm) when going from compound 4 to compound 1 (Table 3). The same trends were also obtained at the experimental level (Table 1), thus 
evidencing that $(E)$ isomers are more affected by inter-branch coupling with respect to $(Z)$ isomers. The delocalization of the orbitals evidenced in Figure S 39 suggests that the photo-excitations are initially delocalized over the three branches and only get localized over a single unit upon nuclear relaxation in the excited state due to the weak spatial overlap (and hence electronic coupling) between the three units. Looking at the energy difference between the $Z$ and $E$ isomerization, we find that this quantity is the same $(0.65 \mathrm{eV})$ for all compounds (Table 3$)$, thus corroborating the similar values of the activation energies estimated by Eyring analysis (see Table 2).

Table 3. DFT calculated properties of compounds 1-6 in their different isomeric forms.

\begin{tabular}{|c|c|c|c|c|c|c|c|}
\hline & & \multirow{2}{*}{$\vartheta\left[{ }^{0}\right]^{a}$} & \multirow{2}{*}{$\begin{array}{l}\Delta E_{\mathrm{Z} \rightarrow \mathrm{E}} \\
{[\mathrm{eV}]^{\mathrm{b}}}\end{array}$} & \multirow{2}{*}{$\begin{array}{c}E_{g} \\
{[\mathrm{eV}]^{\mathrm{c}}}\end{array}$} & \multicolumn{3}{|c|}{$\lambda_{\max }[\mathrm{nm}](f)^{\mathrm{d}}$} \\
\hline & & & & & $\pi-\pi^{*}$ PE & $\pi-\pi^{*}$ Azo & $n-\pi^{*}$ Azo \\
\hline \multirow{4}{*}{1} & $(E, E, E)$ & 38.4 & -1.97 & 3.47 & - & $396(1.52)$ & - \\
\hline & $(E, E, Z)$ & 37.7 & -1.31 & 3.24 & - & $394(1.41)$ & $502(0.12)$ \\
\hline & $(E, Z, Z)$ & 37.8 & -0.65 & 3.24 & - & $378(0.95)$ & $504(0.15)$ \\
\hline & $(Z, Z, Z)$ & 37.9 & 0 & 3.48 & - & - & $504(0.15)$ \\
\hline \multirow{3}{*}{2} & $(E, E)$ & 37.7 & -1.30 & 3.2 & 365 (1.09) & 395 (1.39) & - \\
\hline & $(E, Z)$ & 37.7 & -0.65 & 3.22 & $348(0.97)$ & $380(1.64)$ & $502(0.12)$ \\
\hline & $(Z, Z)$ & 37.8 & 0 & 3.43 & $352(1.64)$ & - & $504(0.14)$ \\
\hline \multirow{2}{*}{3} & $(E)$ & 37.7 & -0.65 & 3.18 & $358(2.47)$ & $382(1.8)$ & - \\
\hline & $(Z)$ & 38 & 0 & 3.43 & $358(2.22)$ & - & $502(0.11)$ \\
\hline \multirow{2}{*}{4} & $(E)$ & 37 & -0.65 & 3.47 & - & $385(1.16)$ & - \\
\hline & (Z) & 36.8 & 0 & 3.51 & - & - & $500(0.09)$ \\
\hline 5 & - & 38.2 & - & 3.79 & $361(2.14)$ & - & - \\
\hline \multirow{2}{*}{6} & (E) & - & -0.64 & 3.78 & - & $342(0.93)$ & \\
\hline & (Z) & - & 0 & 3.66 & - & - & $490(0.05)$ \\
\hline
\end{tabular}

${ }^{a}$ Average dihedral angle formed by the central phenyl ring and the adjacent phenyl ring in the chromophores, as represented in Figure $S$ $37^{\mathrm{b}}$ Relative energy calculated as the difference in energies with respect to the full $(Z)$ isomers. ${ }^{\mathrm{c}}$ Electronic gap determined as the difference between the HOMO-LUMO energies. ${ }^{\mathrm{d}} \lambda_{\max }(\mathrm{nm})$ and oscillator strength ( $\mathrm{f}$ ) computed for the lowest excited state associated to the 1,2-diphenylethyne unit ( $\left.\pi-\pi^{*} \mathrm{PE}\right)$ and the azobenzene arm in the $E\left(\pi-\pi^{*}\right.$ Azo) and $Z\left(n-\pi^{*}\right.$ Azo) forms; note that there might be other closely-lying excited states associated to one arm not discussed here.

\section{Ion mobility mass spectrometry (IMMS)}

The conformational rigidity of our compounds 1-3, combined with the electronic decoupling of the various azobenzenes grafted to the central benzene core, motivated us to probe their shape variation resulting from the $E-Z$ isomerization of their photochromic units. Towards this end, we used ion mobility mass spectrometry (IMMS) to discriminate the different molecular configurations, since this technique allows to resolve mixtures of isomeric ions based on the differences in collisional cross sections (CCS), a property directly related to the gaseous ion geometries. When exposed to ion mobility separation, ions drift under the influence of an electric field and undergo collisions with a buffer gas, typically nitrogen or helium. The ions drift time across the mobility cell is associated to the probability of experiencing collisions with the buffer gas, hence is directly proportional to the ion CCS. ${ }^{18 a}$, 27 Nevertheless, compared to the conventional methods, ionized species are mandatory for conducting IMMS experiments. For the present study, compounds 1-3 bearing carboxylic acid function can be readily deprotonated upon Electrospray lonization (ESI) in the negative ion mode. The mass spectra obtained upon ESI of these star-shaped azobenzene derivatives 1-3 present intense signals, with the most intense peak corresponding to the mono-deprotonated species: $\left[\mathbf{1}-\mathrm{H}^{+}\right]^{-}$at $m / z 749.2,[\mathbf{2}$ $\left.-\mathrm{H}^{+}\right]^{-}$at $m / z 745.2$ and $\left[3-\mathrm{H}^{+}\right]^{-}$at $m / z 741.2$ (Figure S63). Multiply charged ions are also detected, but for our discussion we will only focus on the singly charged ones. When subjected to ion mobility, the singly charged ions $\left[\mathbf{1}-\mathrm{H}^{+}\right]^{-},\left[\mathbf{2}-\mathrm{H}^{+}\right]^{-}$ and $\left[3-\mathrm{H}^{+}\right]^{-}$from non-irradiated solutions, are all characterized by monomodal Arrival Time Distributions (ATD) associated to the all-(E) species. On the other hand, when analyzing solutions irradiated at the UV - PSS, it is striking to monitor the appearance of additional signals at lower drift times (Figure 3). The latter observation clearly enlightens that the UV-generated configurations present more compact geometries compared to the all-E isomer. 
Furthermore, it is worth mentioning that for the star-shaped compounds studied here, the number of signals observed in the ATDs after irradiation corresponds to the number of different configurations, with four, three and two peaks resolved for respectively 1, 2 and 3. The drift times are then used to estimate the experimental collisional cross section, i.e., $C C S_{\text {exp }}$ through a calibration procedure. ${ }^{28}$ Again, the CCS values confirm that the $E$ to $Z$ isomerization induces the compaction of the ion structures with, for instance, $C C S_{\text {exp }}$ drastically decreasing from 269 to $187 \AA^{2}$ when passing from the $(E, E, E)$ to the $(Z, Z, Z)$ configurations (Table 4$)$ of 1 . Interestingly, as a reference experiment, we exposed to UV irradiation and analyzed by IMMS the reference linear azobenzene 4 . The $(Z)$ and $(E)$ configurations could not be resolved with our IMMS instrument when comparing the ATDs of the irradiated and the non-irradiated solutions, although the photoisomerization reaction is attested by UV-Vis measurements (Figure 2 d). This indicates that the large molecular shape rearrangements that are detected for the star-shaped compounds arise from their embedment into large and rigid $\pi$-conjugated scaffolds.

Table 4. Collisional cross sections of the various isomers of 1-3 determined by IMMS.

\begin{tabular}{clllll} 
& $\operatorname{CCS}\left(\AA^{2}\right)$ & \multicolumn{3}{c}{$\operatorname{CCS}\left(\AA^{2}\right)$} & $\operatorname{CCS}\left(\AA^{2}\right)$ \\
\hline$(E, E, E)-1$ & 269 & $(E, E)-2$ & 273 & $(E)-3$ & 277 \\
$(E, E, Z)-1$ & 237 & $(E, Z)-2$ & 239 & $(Z)-3$ & 241 \\
$(E, Z, Z)-1$ & 220 & $(Z, Z)-2$ & 224 & & \\
$(Z, Z, Z)-1$ & 187 & & & &
\end{tabular}

a)

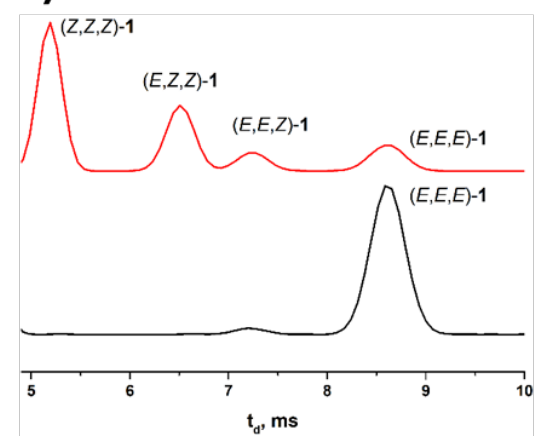

b)

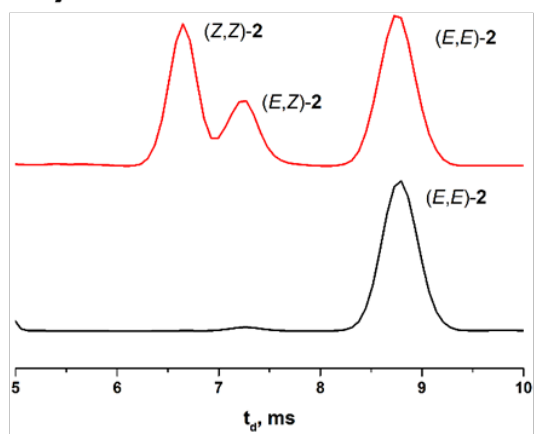

c)

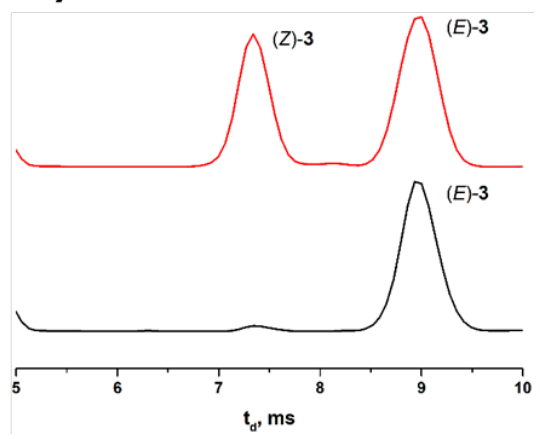

Figure 3. IMMS experiments on compounds 1-3 upon UV light irradiation in solution, Arrival Time Distributions (ATD) recorded for: a) tris(azobenzene) 1, b) bis(azobenzene) $\mathbf{2}$ and c) mono(azobenzene) 3. Black line no light irradiation, red line UV PSS.

To cast further light onto the photoisomerization detected by IMMS and in particular to provide evidence for the stepwise nature of the photoisomerization of $\mathbf{1}$, we followed the light-induced process upon on-line irradiation of a DMSO solution with continuous infusion in the ESI source (Figure 4). We observed a gradual appearance of the aforementioned photogenerated peaks relative to the $(E, E, Z)-\mathbf{1},(E, Z, Z)-\mathbf{1}$ and $(Z, Z, Z)-\mathbf{1}$ isomers, until the UV - PSS is reached. Interestingly, upon integration of the peaks obtained for the four isomers at the UV - PSS, we measured the following apparent isomeric ratio: $63 \%(Z, Z, Z)-\mathbf{1}, 30 \%(E, Z, Z)-\mathbf{1}, 3 \%(E, E, Z)-\mathbf{1}, 4 \%(E, E, E)-\mathbf{1}$. The discrepancy between the IMMS and HPLC-MS data (Table 1) comes from the fact that IMMS data are extracted for singly deprotonated ions while the HPLC-MS quantification is performed for all charge states and possible adducts. 

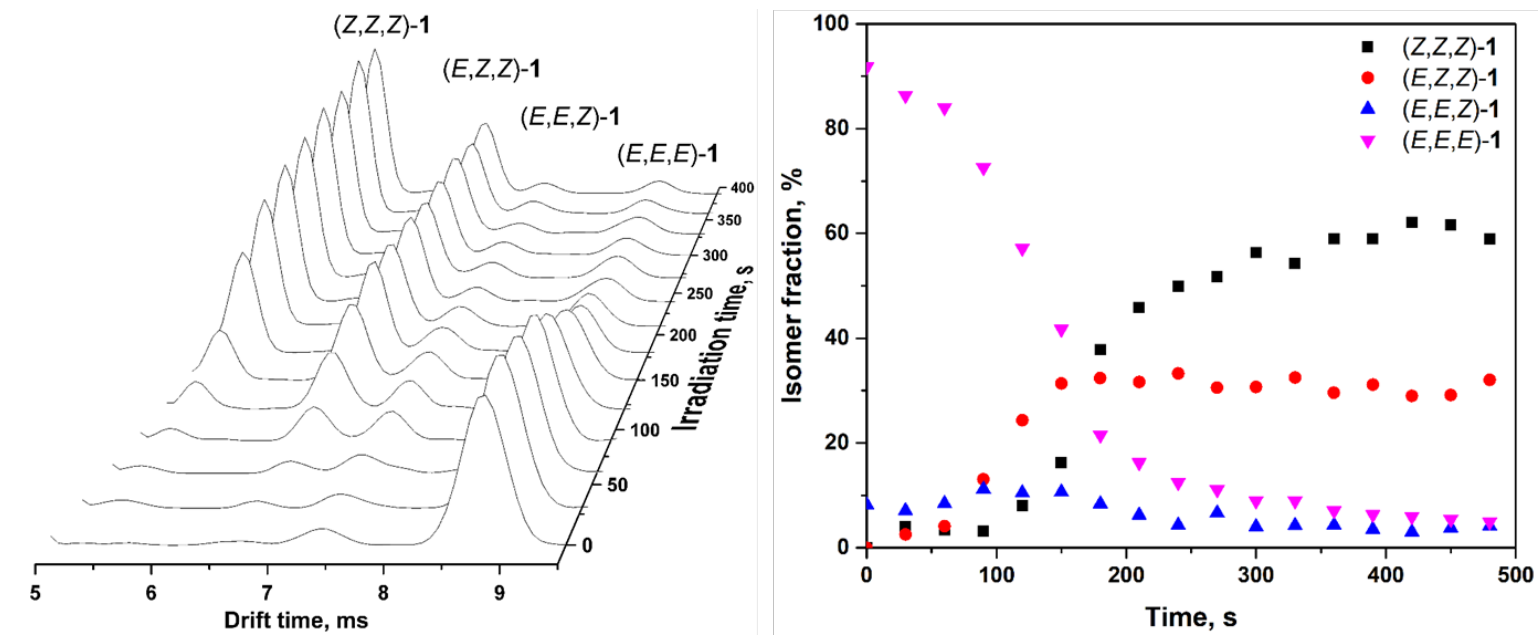

Figure 4. Photoisomerization of $\mathbf{1}$ followed by IMMS. A solution of $\mathbf{1}$ was irradiated with UV light upon IMMS separation. Left, temporal evolution of the arrival time distributions. Right, isomer fraction over time determined by integration of the IMMS signals.
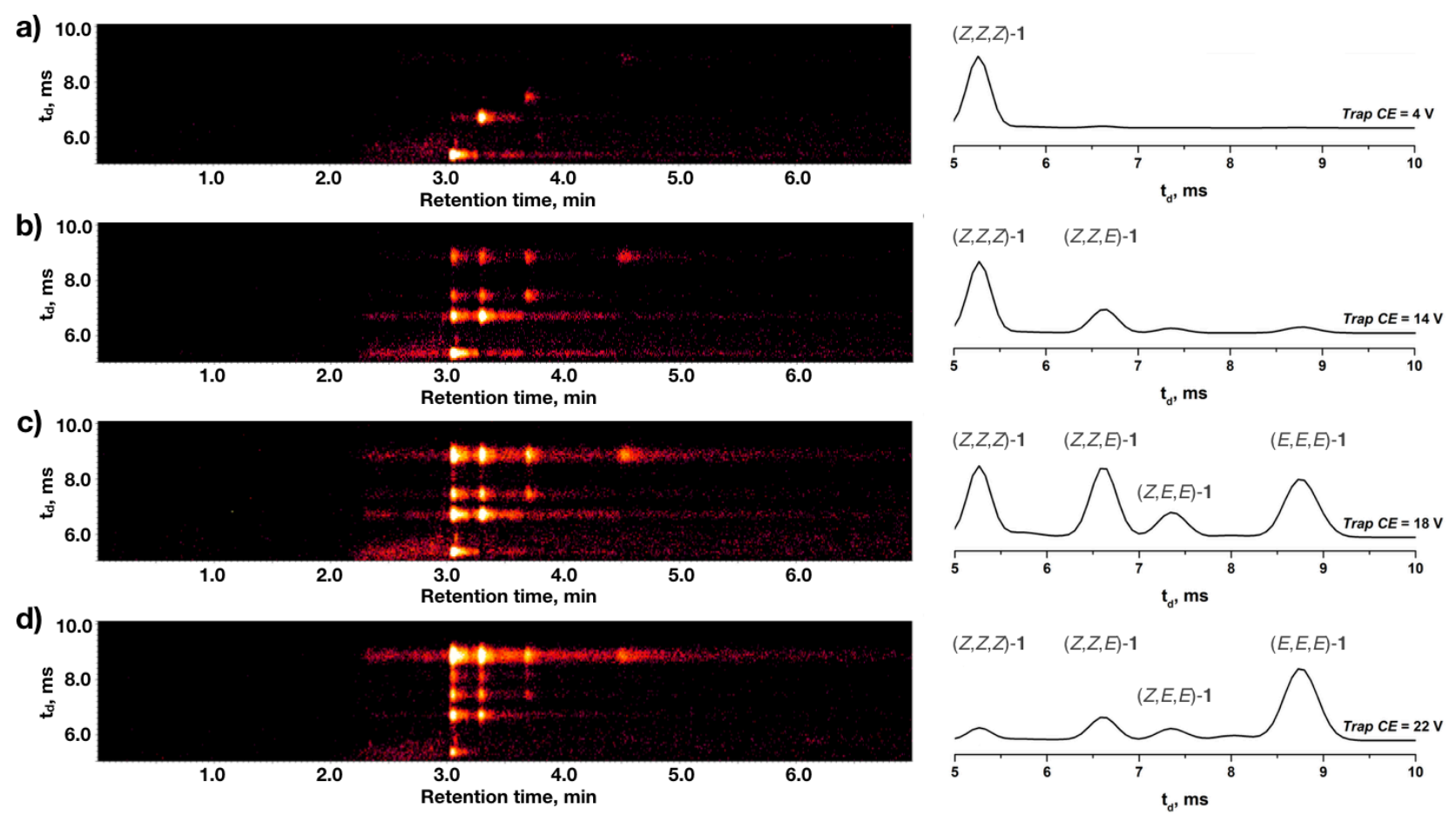

Figure 5. Collisionally-induced $Z \rightarrow E$ isomerization of the $(Z, Z, Z)-1$ ions at different collision energies (Trap CE). a) Trap CE = $4 \mathrm{~V} ; \mathrm{b})$

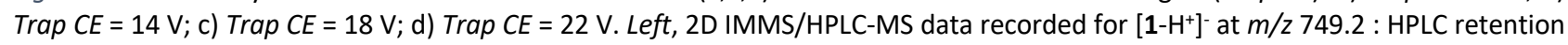
time reported on $x$ axis, ion mobility drift time on $y$ axis. . Signal at $3 \mathrm{~min} 5 \mathrm{~s}$ retention time corresponds to $(Z, Z, Z)-1,3 \mathrm{~min} 20 \mathrm{~s}$ $(Z, Z, E)-\mathbf{1}, 3 \mathrm{~min} 40 \mathrm{~s}(Z, E, E)-\mathbf{1}, 4 \mathrm{~min} 30 \mathrm{~s}(E, E, E)-\mathbf{1}$. Right, Arrival time distributions recorded at various Trap CE for the HPLC signal appearing at $3 \mathrm{~min} 5 \mathrm{~s}$ and corresponding to $(Z, Z, Z)-\mathbf{1}$.

Finally, we investigated the possibility to induce the in-flight $Z \rightarrow E$ isomerization of azobenzene ions by collisional activation, prior to their separation by ion mobility. ${ }^{18 a}$ In this case, we employed an HPLC set-up to separate the four isomers before injection in the mass spectrometer and performed the aforementioned experiments on the isolated photogenerated $(Z, Z, Z)-\mathbf{1}$ isomer. Such an experiment benefits from the great versatility of ion manipulations offered by the Waters Synapt G2-Si mass spectrometer. After HPLC separation of the isomers of 1 and the preparation of the gas phase ions upon ESI(-), the molecular anions ([1 $\left.-\mathrm{H}^{+}\right]^{-}$at $m / z$ 749.2) are mass-selected with the quadrupole mass selector (see SI for further experimental details). The ions are then subjected to collisional heating (collisional activation) prior to the ion mobility separation by gradually increasing their kinetic energy within the Trap cell (Figure 5 , figure S64). It is worth noting that we only used low voltages (Trap CE) to induce isomerization and avoid ion decomposition. At default Trap CE (4 V), only the signature of the $(Z, Z, Z)$ ions is detected upon IMMS, demonstrating the successful isolation of the corresponding species by HPLC separation. This also reveals that no isomerization is 
induced in source or in the ion transfer regions with our experimental setup. Upon progressive increase of the Trap $C E$, additional signals are detected in the ATDs and are related to the progressive appearance of the $E$ isomers. Interestingly, the successive appearance of the $(E, Z, Z),(E, E, Z)$ and finally $(E, E, E)$ isomers also reveals that the thermal isomerization of the $(Z, Z, Z)$ ions is a stepwise process. These experimental evidences suggest that upon collisional activation, in analogous fashion as heating, it is possible to induce the thermal $Z \rightarrow E$ isomerization of azobenzenes, and hence to generate the more thermodynamically stable $E$ isomer(s) within the timescale $(\mu \mathrm{s})$ of their residence in the Trap Cell prior to the ion mobility separation.

\section{CONCLUSIONS}

In summary, we have designed and synthesized a novel family of multi-azobenzene compounds by connecting individual photochromes to a central trisubstituted 1,3,5-benzene core. Such star-shaped systems consist of conformationally rigid, pseudo-planar scaffolds. The unique design in our tris-, bis- and mono(azobenzene) compounds limits the $\pi$-conjugation between the switches belonging to the same molecule, thus enabling their efficient isomerization and the independent behavior of each unit with respect to the state of the neighboring units, while maintaining a high structural rigidity. An in-depth experimental insight has been attained by making use of different complementary techniques such as UV-Vis absorption spectroscopy, cyclic voltammetry, differential pulse voltammetry, high performance liquid chromatography and advanced mass spectrometry methods such as ion mobility. The additivity of the UV-Vis absorption spectra relative to the all-(E) isomer, together with the appearance of clear isosbestic points upon photoswitching of mono-, bis- and tris(azobenzene) compounds provided unambiguous evidences for a weak coupling between the chromophores. This was further shown by cyclic and differential pulse voltammetry, evidencing that the reduction of the azobenzene units within the multi(azobenzene) compounds occur at the same potential. The weak electronic coupling between the branches allows for a remarkably efficient photoswitching of all azobenzenes, as evidenced by their photochemical quantum yields, not substantially different from the values shown by reference mono-azobenzenes. Moreover, all star-shaped compounds showed Z-rich UV photostationary states. Ion mobility mass spectrometry was exploited to study multi-photochromic compounds revealing the occurrence of a large molecular shape change in our rigid star-shaped azobenzene derivatives. While the combination of conventional photophysical studies with mass spectrometry-related techniques such as IMMS shall be used in the future to single out the photoswitching properties of multi-chromophoric arrays, we envisage that our $C_{3}$-symmetrical star-shaped multiphotochromic design enables to develop a host of complex stimuli-responsive systems, in particular for the realization of light-triggered porous materials such as metal-organic-, or covalent-organic frameworks.

\section{ASSOCIATED CONTENT}

The Supporting Information is available free of charge via the Internet at: www.------.com

Detailed experimental procedures, synthesis and characterization of the products, computational methodologies.

\section{AUTHOR INFORMATION}

\section{Corresponding Authors}

*samori@unistra.fr, *marcel.mayor@unibas.ch, *pascal.gerbaux@umons.ac.be, *jerome.cornil@umons.ac.be

\section{Author Contributions}

${ }^{\dagger}$ These authors contributed equally.

\section{ACKNOWLEDGEMENTS}

We gratefully thank Dr. Sara Bonacchi, Dr. Martin Herder and Dr. Stefano Casalini for enlightening discussions. This work was supported by the EC through the Marie Sklodowska-Curie ITN project iSwitch (GA-642196), the Agence 
Nationale de la Recherche through the LabEx project Chemistry of Complex Systems (ANR-10-LABX-0026_CSC) and the International Center for Frontier Research in Chemistry (icFRC). M.V. and M.M. acknowledge financial support by the Helmholtz Research Programm STN (Science and Technology of Nanosystems). Computational resources were provided by the Consortium des Équipements de Calcul Intensif (CÉCl) funded by the Belgian National Fund for Scientific Research (F.R.S.-FNRS) under Grant 2.5020.11. Q.D. and J.C. are FNRS research fellows.

\section{REFERENCES}

(1) (a) Feringa, B. L. The Art of Building Small: From Molecular Switches to Motors (Nobel Lecture). Angew. Chem., Int. Ed. 2017, 56 (37), 11060-11078; (b) Sauvage, J. P. From Chemical Topology to Molecular Machines (Nobel Lecture). Angew. Chem., Int. Ed. 2017, 56 (37), 11080-11093; (c) Stoddart, J. F. Mechanically Interlocked Molecules (MIMs)-Molecular Shuttles, Switches, and Machines (Nobel Lecture). Angew. Chem., Int. Ed. 2017, 56 (37), 11094-11125.

(2) (a) Molecular Switches. (Ed.: Feringa, B. L.), Wiley-VCH, Weinheim, 2001; (b) Balzani, V.; Credi, A.; Raymo, F. M.; Stoddart, J. F. Artificial Molecular Machines. Angew. Chem., Int. Ed. 2000, 39 (19), 3348-3391; ; (c) Kinbara, K.; Aida, T. Toward Intelligent Molecular Machines: Directed Motions of Biological and Artificial Molecules and Assemblies. Chem. Rev. 2005, 105 (4), 1377-1400; (d) ErbasCakmak, S.; Leigh, D. A.; McTernan, C. T.; Nussbaumer, A. L. Artificial Molecular Machines. Chem. Rev. 2015, 115 (18), 10081-10206; (e) Kassem, S.; van Leeuwen, T.; Lubbe, A. S.; Wilson, M. R.; Feringa, B. L.; Leigh, D. A. Artificial Molecular Motors. Chem. Soc. Rev. 2017, 46 (9), 2592-2621; (f) Leigh, D. A. Genesis of the Nanomachines: The 2016 Nobel Prize in Chemistry. Angew. Chem., Int. Ed. 2016, 55 (47), 14506-14508.

(3) (a) Lerch, M. M.; Hansen, M. J.; Velema, W. A.; Szymanski, W.; Feringa, B. L. Orthogonal Photoswitching in a Multifunctional Molecular System. Nat. Commun. 2016, 7, 12054; (b) Zhao, F.; Grubert, L.; Hecht, S.; Bléger, D. Orthogonal Switching in Four-State Azobenzene Mixed-Dimers. Chem. Commun. 2017, 53 (23), 3323-3326; (c) Manna, D.; Udayabhaskararao, T.; Zhao, H.; Klajn, R. Orthogonal Light-Induced Self-Assembly of Nanoparticles using Differently Substituted Azobenzenes. Angew. Chem., Int. Ed. 2015, 54 (42), 12394-12397.

(4) (a) Russew, M. M.; Hecht, S. Photoswitches: from Molecules to Materials. Adv. Mater. 2010, 22 (31), 3348-3360; (b) Saha, S.; Stoddart, J. F. Photo-Driven Molecular Devices. Chem. Soc. Rev. 2007, 36 (1), 77-92; (c) Ceroni, P.; Credi, A.; Venturi, M.; Balzani, V. Light-Powered Molecular Devices and Machines. Photochem. Photobiol. Sci. 2010, 9 (12), 1561-1573; (d) Brieke, C.; Rohrbach, F.; Gottschalk, A.; Mayer, G.; Heckel, A. Light-Controlled Tools. Angew. Chem., Int. Ed. 2012, 51 (34), 8446-8476; (e) Balzani, V.; Credi, A.; Venturi, M. Light powered molecular machines. Chem. Soc. Rev. 2009, 38 (6), 1542-1550.

(5) (a) Zhao, W. L.; Carreira, E. M. A Smart Photochromophore through Synergistic Coupling of Photochromic Subunits. J. Am. Chem. Soc. 2002, 124 (8), 1582-1583; (b) Norikane, Y.; Tamaoki, N. Light-Driven Molecular hinge: A New Molecular Machine Showing a Light-Intensity-Dependent Photoresponse that Utilizes the Trans-Cis Isomerization of Azobenzene. Org. Lett. 2004, 6 (15), 2595-2598; (c) Higashiguchi, K.; Matsuda, K.; Tanifuji, N.; Irie, M. Full-Color Photochromism of a Fused Dithienylethene Trimer. J. Am. Chem. Soc. 2005, 127 (25), 8922-8923; (d) Higashiguchi, K.; Matsuda, K.; Irie, M. Photochromic Reaction of a Fused Dithienylethene: Multicolor Photochromism. Angew. Chem., Int. Ed. 2003, 42 (30), 3537-3540; (e) Andréasson, J.; Pischel, U.; Straight, S. D.; Moore, T. A.; Moore, A. L.; Gust, D. All-Photonic Multifunctional Molecular Logic Device. J. Am. Chem. Soc. 2011, 133 (30), 11641-11648; (f) Frey, J.; Kodis, G.; Straight, S. D.; Moore, T. A.; Moore, A. L.; Gust, D. Photonic Modulation of Electron Transfer with Switchable Phase Inversion. J. Phys. Chem. A 2013, 117 (3), 607-615; (g) Yonekawa, I.; Mutoh, K.; Kobayashi, Y.; Abe, J. Intensity-Dependent Photoresponse of Biphotochromic Molecule Composed of a Negative and a Positive Photochromic Unit. J. Am. Chem. Soc. 2018, 140 (3), 1091-1097.

(6) (a) Fihey, A.; Perrier, A.; Browne, W. R.; Jacquemin, D. Multiphotochromic Molecular Systems. Chem. Soc. Rev. 2015, 44 (11), 3719-3759; (b) Perrier, A.; Maurel, F.; Jacquemin, D. Single Molecule Multiphotochromism with Diarylethenes. Acc. Chem. Res. 2012, 45 (8), 1173-1182. 
(7) (a) Li, C.; Yan, H.; Zhao, L. X.; Zhang, G. F.; Hu, Z.; Huang, Z. L.; Zhu, M. Q. A Trident DithienylethenePerylenemonoimide Dyad with Super Fluorescence Switching Speed and Ratio. Nat. Commun. 2014, 5, 5709; (b) Biellmann, T.; Galanti, A.; Boixel, J.; Wytko, J. A.; Guerchais, V.; Samorì, P.; Weiss, J. Fluorescence Commutation and Surface Photopatterning with Porphyrin Tetradithienylethene Switches. Chem. Eur. J. 2018, 24 (7), 1631-1639.

(8) Fihey, A.; Russo, R.; Cupellini, L.; Jacquemin, D.; Mennucci, B. Is Energy Transfer Limiting Multiphotochromism? Answers from Ab Initio Quantifications. Phys. Chem. Chem. Phys. 2017, 19 (3), 2044-2052.

(9) (a) Griffiths, J. II. Photochemistry of Azobenzene and its Derivatives. Chem. Soc. Rev. 1972, 1 (4), 481-493; (b) Beharry, A. A.; Woolley, G. A. Azobenzene Photoswitches for Biomolecules. Chem. Soc. Rev. 2011, 40 (8), 4422-4437; (c) Bullock, D. J. W.; Cumper, C. W. N.; Vogel, A. I. 989. Physical Properties and Chemical Constitution. Part XLIII. The Electric Dipole Moments of Azobenzene, Azopyridines, and Azoquinolines. J. Chem. Soc. 1965, 5316-5323.

(10) Bléger, D.; Yu, Z.; Hecht, S. Toward Optomechanics: Maximizing the Photodeformation of Individual Molecules. Chem. Commun. 2011, 47 (45), 12260-12266.

(11) (a) Bléger, D.; Dokić, J.; Peters, M. V.; Grubert, L.; Saalfrank, P.; Hecht, S. Electronic Decoupling Approach to Quantitative Photoswitching in Linear Multiazobenzene Architectures. J. Phys. Chem. B 2011, 115 (33), 9930-9940; (b) Cisnetti, F.; Ballardini, R.; Credi, A.; Gandolfi, M. T.; Masiero, S.; Negri, F.; Pieraccini, S.; Spada, G. P. Photochemical and Electronic Properties of Conjugated Bis(Azo) Compounds: an Experimental and Computational Study. Chem. Eur. J. 2004, 10 (8), 2011-2021.

(12) (a) Bahrenburg, J.; Sievers, C. M.; Schönborn, J. B.; Hartke, B.; Renth, F.; Temps, F.; Näther, C.; Sönnichsen, F. D. Photochemical Properties of Multi-Azobenzene Compounds. Photochem. Photobiol. Sci. 2013, 12 (3), 511-518; (b) Lee, S.; Oh, S.; Lee, J.; Malpani, Y.; Jung, Y. S.; Kang, B.; Lee, J. Y.; Ozasa, K.; Isoshima, T.; Lee, S. Y.; Hara, M.; Hashizume, D.; Kim, J. M. Stimulus-Responsive Azobenzene Supramolecules: Fibers, Gels, and Hollow Spheres. Langmuir 2013, 29 (19), 5869-5877; (c) Kind, J.; Kaltschnee, L.; Leyendecker, M.; Thiele, C. M. Distinction of Trans-Cis Photoisomers with Comparable Optical Properties in Multiple-State Photochromic Systems - Examining a Molecule with Three Azobenzenes via In Situ Irradiation NMR Spectroscopy. Chem. Commun. 2016, 52 (84), 12506-12509; (d) Koch, M.; Saphiannikova, M.; Santer, S.; Guskova, O. Photoisomers of Azobenzene Star with a Flat Core: Theoretical Insights into Multiple States from DFT and MD Perspective. J. Phys. Chem. B 2017, 121 (37), 8854-8867.

(13) Segarra-Maset, M. D.; van Leeuwen, P. W. N. M.; Freixa, Z. Light Switches the Ligand! Photochromic Azobenzene-Phosphanes. Eur. J. Inorg. Chem. 2010, 2010 (14), 2075-2078.

(14) (a) Bléger, D.; Liebig, T.; Thiermann, R.; Maskos, M.; Rabe, J. P.; Hecht, S. Light-Orchestrated Macromolecular "Accordions": Reversible Photoinduced Shrinking of Rigid-Rod Polymers. Angew. Chem., Int. Ed. 2011, 50 (52), 12559-12563; (b) Yu, Z.; Hecht, S. Reversible and Quantitative Denaturation of Amphiphilic Oligo(Azobenzene) Foldamers. Angew. Chem., Int. Ed. 2011, 50 (7), 1640-1643.

(15) (a) Côté, A. P.; El-Kaderi, H. M.; Furukawa, H.; Hunt, J. R.; Yaghi, O. M. Reticular Synthesis of Microporous and Mesoporous 2D Covalent Organic Frameworks. J. Am. Chem. Soc. 2007, 129 (43), 12914-12915; (b) Ghosh, K.; Hu, J.; White, H. S.; Stang, P. J. Construction of Multifunctional Cuboctahedra via Coordination-Driven Self-Assembly. J. Am. Chem. Soc. 2009, 131 (19), 6695-6697; (c) Furukawa, H.; Ko, N.; Go, Y. B.; Aratani, N.; Choi, S. B.; Choi, E.; Yazaydin, A. O.; Snurr, R. Q.; O'Keeffe, M.; Kim, J.; Yaghi, O. M. Ultrahigh Porosity in Metal-Organic Frameworks. Science 2010, 329 (5990), 424-428; (d) Vijayaraghavan, S.; Ecija, D.; Auwarter, W.; Joshi, S.; Seufert, K.; Drach, M.; Nieckarz, D.; Szabelski, P.; Aurisicchio, C.; Bonifazi, D.; Barth, J. V. Supramolecular Assembly of Interfacial Nanoporous Networks with Simultaneous Expression of Metal-Organic and OrganicBonding Motifs. Chem. Eur. J. 2013, 19 (42), 14143-14150.

(16) Galanti, A.; Diez-Cabanes, V.; Santoro, J.; Valášek, M.; Minoia, A.; Mayor, M.; Cornil, J.; Samorì, P. Electronic Decoupling in C3-Symmetrical Light-Responsive Tris(Azobenzene) Scaffolds: SelfAssembly and Multiphotochromism. J. Am. Chem. Soc. 2018, 140 (47), 16062-16070. 
(17) (a) Kolotuchin, S. V.; Fenlon, E. E.; Wilson, S. R.; Loweth, C. J.; Zimmerman, S. C. Self-Assembly of 1,3,5-Benzenetricarboxylic Acids(Trimesic Acids) and Several Analogues in the Solid State. Angew. Chem., Int. Ed. 1996, 34 (2324), 2654-2657; (b) Dienstmaier, J. F.; Mahata, K.; Walch, H.; Heckl, W. M.; Schmittel, M.; Lackinger, M. On the scalability of supramolecular networks - high packing density vs optimized hydrogen bonds in tricarboxylic acid monolayers. Langmuir 2010, 26 (13), 10708-10716.

(18) (a) Révész, Á.; Schröder, D.; Rokob, T. A.; Havlík, M.; Dolenský, B. In-Flight Epimerization of a BisTröger Base. Angew. Chem., Int. Ed. 2011, 50 (10), 2401-2404; (b) Thiel, J.; Yang, D.; Rosnes, M. H.; Liu, X.; Yvon, C.; Kelly, S. E.; Song, Y. F.; Long, D. L.; Cronin, L. Observing the Hierarchical SelfAssembly and Architectural Bistability of Hybrid Molecular Metal Oxides Using Ion-Mobility Mass Spectrometry. Angew. Chem., Int. Ed. 2011, 50 (38), 8871-8875; (c) Santos, J. J.; Toma, S. H.; Lalli, P. M.; Riccio, M. F.; Eberlin, M. N.; Toma, H. E.; Araki, K. Exploring the coordination chemistry of isomerizable terpyridine derivatives for successful analyses of cis and trans isomers by travelling wave ion mobility mass spectrometry. Analyst 2012, 137 (17), 4045-4051; (d) Urner, L. H.; Thota, B. N.; Nachtigall, O.; Warnke, S.; von Helden, G.; Haag, R.; Pagel, K. Online Monitoring the Isomerization of an Azobenzene-Based Dendritic Bolaamphiphile Using Ion Mobility-Mass Spectrometry. Chem. Commun. 2015, 51 (42), 8801-8804; (e) Bull, J. N.; Scholz, M. S.; Coughlan, N. J.; Kawai, A.; Bieske, E. J. Monitoring Isomerization of Molecules in Solution Using Ion Mobility Mass Spectrometry. Anal. Chem. 2016, 88 (24), 11978-11981.

(19) Yamaguchi, Y.; Ochi, T.; Miyamura, S.; Tanaka, T.; Kobayashi, S.; Wakamiya, T.; Matsubara, Y.; Yoshida, Z. Rigid Molecular Architectures that Comprise a 1,3,5-Trisubstituted Benzene Core and Three Oligoaryleneethynylene Arms: Light-Emitting Characteristics and Pi Conjugation Between the Arms. J. Am. Chem. Soc. 2006, 128 (14), 4504-4505.

(20) Archut, A.; Vögtle, F.; De Cola, L.; Azzellini, G. C.; Balzani, V.; Ramanujam, P. S.; Berg, R. H. Azobenzene-Functionalized Cascade Molecules: Photoswitchable Supramolecular Systems. Chem. Eur. J. 1998, 4 (4), 699-706.

(21) Kuhn, H. J.; Braslavsky, S. E.; Schmidt, R. Chemical Actinometry. Pure Appl. Chem. 2004, 76 (12), 2105-2146.

(22) Gauglitz, G.; Hubig, S. Chemical Actinometry in the UV by Azobenzene in Concentrated Solution: A Convenient Method. J. Photochem. 1985, 30 (2), 121-125.

(23) Rau, H., In Photochromism, Molecules and Systems (Ed.: Dürr, H.; Bouas-Laurent, H.), Elsevier, Amsterdam, 1990; chapter 4.

(24) (a) Aylward, G. H.; Garnett, J. L.; Sharp, J. H. Alternating and direct current polarography of azobenzene in indifferent electrolyte in dimethylformamide. Anal. Chem. 1967, 39 (4), 457-460; (b) Sadler, J. L.; Bard, A. J. Electrochemical reduction of aromatic azo compounds. J. Am. Chem. Soc. 1968, 90 (8), 1979-1989.

(25) Connors, K. A., Chemical Kinetics: the Study of Reaction Rates in Solution. (Ed.: Sons, J. W.), 1990.

(26) Dokić, J.; Gothe, M.; Wirth, J.; Peters, M. V.; Schwarz, J.; Hecht, S.; Saalfrank, P. Quantum Chemical Investigation of Thermal Cis-to-Trans Isomerization of Azobenzene Derivatives: Substituent Effects, Solvent Effects, and Comparison to Experimental Data. J. Phys. Chem. A 2009, 113 (24), 6763-6773. Lanucara, F.; Holman, S. W.; Gray, C. J.; Eyers, C. E. The power of ion mobility-mass spectrometry for structural characterization and the study of conformational dynamics. Nat. Chem. 2014, 6 (4), 281-294.

(28) Duez, Q.; Chirot, F.; Lienard, R.; Josse, T.; Choi, C.; Coulembier, O.; Dugourd, P.; Cornil, J.; Gerbaux, P.; De Winter, J. Polymers for Traveling Wave Ion Mobility Spectrometry Calibration. J. Am. Soc. Mass. Spectrom. 2017, 28 (11), 2483-2491. 